\title{
Warum gehen die Deutschen so oft zum Arzt?
}

- 18-mal im Jahr wird der Deutsche zum Patienten. Damit sind wir Weltspitze. Was die Leute hierzulande so häufig in eine Arztpraxis treibt, versuchte eine Untersuchung der Technischen Universität München zu klären. Hierzu wurden im vergangenen Jahr Daten von 1005 Patienten aus 13 oberbayerischen Hausarztpraxen ausgewertet.

15,3 Arztbesuche und 3,8 Überweisungen hielten den durchschnittlichen Deutschen im Jahr 2010 auf Trab. An 7,5 Tagen war er arbeitsunfähig. Die Frauen waren unter den Patienten mit 58,5\% leicht in der Überzahl, das Durchschnittsalter lag insgesamt bei 49,3 Jahren. Bei $80,7 \%$ der Ratsuchenden wurde mindestens ein körperliches Leiden diagnostiziert, bei $28,3 \%$ mindestens eine psychische Störung. $24,8 \%$ der Patienten hatten sowohl somatische als auch seelische Probleme. Bei den psychischen Erkrankungen standen an erster Stelle die somatoformen Störungen, dicht gefolgt von Depression und Angsterkrankungen sowie Panikstörungen.

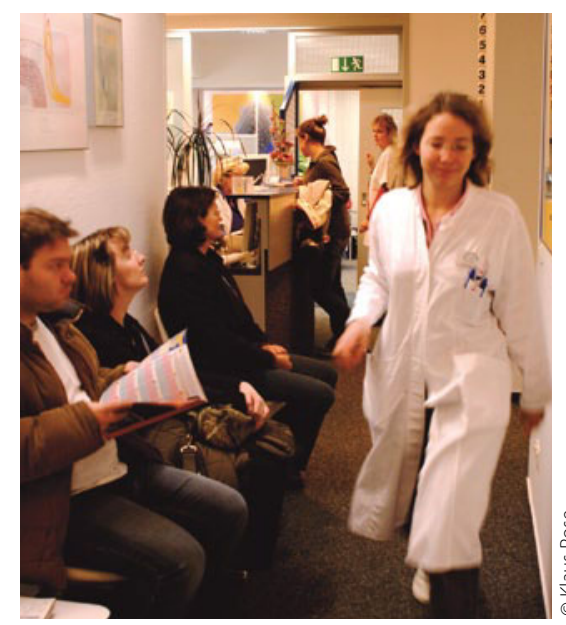

Vor allem Patienten mit psychosomatischen Problemen füllen die Praxen.

Die Daten machen deutlich, dass Patienten mit psychischen Dauerproblemen häufiger einen Arzt aufsuchen als solche mit rein somatischen Erkrankungen (OR 20,0 vs. 14,4). Während körperliche Beschwerden häufiger zu Überweisungen führten (OR 4,9 vs. $3,6)$, machten psychische Erkrankungen ei- ne Arbeitsunfähigkeit wahrscheinlicher als ein körperliches Leiden (OR 5,0 vs. 2,5).

Die Autoren vermuten, dass vor allem die psychosomatischen Komorbiditäten für die vollen Wartezimmer in Deutschlands Hausarztpraxen sowie für die Dauer der Arbeitsunfähigkeit verantwortlich sind. Der oft enge Zeitplan in den Praxen führt dazu, dass die seelischen Probleme der Patienten zu wenig Beachtung finden. So sitzen diese Patienten immer wieder mit ihren ungelösten Problemen in den Wartezimmern. Für die oft komplexen Krankheitsbilder sind die heutzutage üblichen sechs bis zehn Minuten, die dem Hausarzt pro Patient zur Verfügung stehen, zu wenig. So fordern die Autoren strukturelle Änderungen im Vergütungssystem, um es den Ärzten zu erlauben, sich adäquat um die Patienten zu kümmern, die wegen ihrer psychischen Probleme einen höheren Zeitaufwand erfordern.

ST .

Schneider A. et al. Unlimited access to health careimpact of psychosomatic co-morbidity on utilization in German general practices. BMC Family Practice 2011;12:514.7.11

\section{CHRONISCHE VIRUSHEPATITIDEN \\ Eine Frage mehr kann Leben retten}

- Am 28. Juli ist Welt-Hepatitis-Tag: Mit diesem Tag soll auf die hohe Zahl unerkannter Infektionen mit Hepatitis-B- und -C-Viren aufmerksam gemacht werden. In Deutschland leiden Schätzungen zufolge 500000 Menschen an einer chronischen Hepatitis B und 600000 an einer chronischen Hepatitis C. Diagnostiziert ist die Erkrankung nur bei der Hälfte. Unbehandelt können Hepatitis $B$ und $C$ in eine Zirrhose und schließlich in Leberkrebs münden. Diese Spätstadien verursachen in Deutschland pro Jahr mehr als 50000 Todesfälle. Dabei bestehen bei frühem Therapiebeginn bei Hepatitis $C$ gute Heilungschancen, und auch eine Hepatitis B lässt sich kontrollieren.

\section{Die Leber leidet still}

Das Problem: „Die kranke Leber verursacht keine Schmerzen - sie leidet still", sagt Prof. Dr. Claus Niederau von der Deutschen Leberhilfe e. V. Wenn Symptome auftreten, sind sie unspezifisch (Müdigkeit, Abgeschlagenheit, Druck im Oberbauch) und werden meist nicht richtig zugeordnet. Die Deutsche Leberhilfe hat daher die Früherkennung zum zentralen Thema des WeltHepatitis-Tages gemacht. Vor allem Allgemeinmediziner und Internisten sollen dazu beitragen - durch nur "eine Frage mehr", wie auch das Motto der diesjährigen Kampagne lautet. „Wollen wir Ihre Leberwerte gleich mit überprüfen?", heißt die entscheidende Frage im Patientengespräch. Die Be- stimmung von GPT, GOT und GGT kann einen ersten Hinweis auf virale Leberinfektionen liefern.

Die Deutsche Leberhilfe e. V. fordert die Ärzte auf, sich für die Früherkennung stark zu machen und der Kampagne ein Gesicht zu geben - mit Fotos von ihrem Praxisteam auf der Webseite www.einefrage-mehr.de. Zum Abschluss der Aktion sollen die Fotos in einem Kampagnenfilm präsentiert werden. Ziel ist, auch Gesundheitspolitiker von der Brisanz des Themas zu überzeugen und die Aufnahme von Lebertests in den GKVLeistungskatalog zu erwirken. BS =

Informationen zur Kampagne und zur Fotoaktion finden Sie auf www.eine-frage-mehr.de. 